\title{
Knowledge, Perception and Women's Experience on Induction of Labour - A Questionnaire-based observational study in South India
}

\section{Rajeshwari G Bhat}

Melaka Manipal Medical College, Manipal, Manipal Academy of Higher education, Manipal vinutha vinod ( $\nabla$ vinuthakvinod@gmail.com )

MMMC,MANIPAL ACADEMY OF HIGHER EDUCATION https://orcid.org/0000-0001-8768-3251

\section{Parvati V Bhat}

Melaka Manipal Medical College, MANIPAL, Manipal Academy of Higher education, Manipal

\section{Nida Zahoor}

Melaka Manipal Medical College,Manipal, Manipal Academy of Higher education, manipal

\section{Research article}

Keywords: Women's experience, induction of labour, labour pain, spontaneous labour, experience of labour

Posted Date: March 18th, 2021

DOl: https://doi.org/10.21203/rs.3.rs-330028/v1

License: (9) This work is licensed under a Creative Commons Attribution 4.0 International License. Read Full License 


\section{Abstract}

Background Induction of labour is an indispensable obstetric procedure.Very few studies have concentrated on women;s experience of induction of labour. Its been reported that women generally have a negative experience with Induction...There is a lack of data regarding the experience of labour induction among Indian women. We carried out this study womens experience on the induction of labour in this part of South Asia. This study highlights the unmet need of women undergoing induction of labour and changes obstetricians can implement in their day to day practice to make childbirth a positive experience

Methods The aim was to study the Knowledge, Perception and Women's experience on Induction of labour and to compare this labour experience with women who have come in spontaneous labour. During the study period 300 women who were induced, formed the study group, every consecutive women who came in spontaneous labour formed the control group. Validated. Questionnaires were given after delivery for both induced and to women who delivered spontaneously. Ethical clearance was obtained by the institution (MUEC/13/2015-2016) Data were analysed using SPSS 16. Percentage was used to summarize the categorical data, Chi-square test for associations and Independent $t$ test to compare visual analogue scores

Results Out of 300 women who were induced $65 \%(196)$ were aware of induction .84\% (249) women knew the reason for their induction.57\%(170/300) were aware of benefits of induction of labour, whereas only $15 \%(45 / 300)$ were informed about adverse effects .54\% (162/300) of women felt analgesia given was not satisfactory. $61 \%(177)$ of women had positive, and $14 \%(40)$ had negative attitude towards induction.32\%(94)spontaneously labouring women felt labour unbearable compared to $45 \%$ (126) induced women which is statistically significant ( $p$ value 0.001 ). While comparing the two groups, induced women felt more sad $(p<0.001)$ and tired $(p=0.01)$ compared to women in spontaneous group.

Discussion Induced women were not well informed about adverse effects and felt labour was unbearable .Induced women were more tired and sad at the end of labour..Women should be provided with realistic information, and better analgesia in labor

Conclusion Induced women were not well informed about adverse effects and felt labour was unbearable .Induced women were more tired and sad at the end of labour. Women should be offered better analgesia in labour. Good counselling and compassionate care from healthcare workers may increase the satisfaction levels of induced women.

\section{Background}

Induction of labour ( $\mathrm{IOL})$ is defined, as the initiation of uterine contractions in a pregnant woman who is not in labour, to have a vaginal birth within 24 to 48 hours (Leduc et al., 2013) Childbirth is an experience that has the power to transform a woman forever. Induced labour can have an impact on the birth experience and can be more painful and less effective than spontaneous labour (NICE Clinical Guideline 70, 2008). 
In developing countries, the doctor-patient ratio is low resulting in increased workload on the obstetricians. Importance to medical management of the mother outweighs the psychological needs. Time constraints prevent optimum antenatal counselling and education.

Increasing medicalization of childbirth processes negatively impacts the childbirth experience. (Warren et al., 2018) Negative childbirth experience in turn may affect the mother's ability to bond with the newborn, create fear about future childbirth, and may even result in postpartum depression. Women have a right to respectful maternity care and positive childbirth experience. A positive experience is one that fulfils or exceeds her prior personal and socio-cultural beliefs and expectations (Warren et al., 2018).

Most studies on IOL have dealt with methods available and clinical outcomes. The Woman's knowledge, attitude, perceptions and preferences and experience of IOL have not been extensively studied.

Assessment of the needs and experience of pregnant women during induction is essential to identify the support they need and prefer. (NICE Clinical Guideline 70, 2008) Such studies on women's experience are needed to evaluate the quality of maternity services. (Thompson \& Miller, 2014).

Women in spontaneous labour are more satisfied compared to induced women (Shetty et al., 2005) (Hildingsson et al., 2011). However, in one study (Heimstad et al., 2007) induced women were reported to have a positive experience. Women undergoing induction should be provided with quality information and encouraged to be involved in decision making and provided with adequate support (Murtagh \& Folan, 2014). To the best of our knowledge, this questionnaire-based study is the first of its kind in India that aims to analyses the knowledge, attitude, experience of expectant mothers about induction of labour and to compare it with the experience of women in spontaneous labour.

\section{Material And Methods}

This study was conducted at Dr TMA Pai Hospital, Udupi, which is a secondary level teaching hospital affiliated to Manipal Academy Of Higher Education. This was a prospective observational and comparative study done over 15 months.

Pregnancies with a single live foetus in vertex presentation and gestational age more than 36 weeks were included in the study. Pregnancies with antepartum haemorrhage, previous uterine surgeries, congenital anomalies and intrauterine death of the foetus were excluded from the study. There were 1575 deliveries at the institution during the study period, out of

which 550 were induced (Induction Rate of 34\%). During the study period, 300 women who were induced consented to participate in the study and formed the study group. Their outcomes were compared with consecutive women who came in spontaneous labour.Participants in each group answered the questions and there was no missing data.

The study was performed in accordance with the ethical standards laid down in the 1964 Declaration of Helsinki and its later amendments. Ethical clearance was obtained by the ethical committee of the 
institution (MUEC/13/2015-2016). To estimate a $25 \%$ incidence of induced labour at $95 \%$ confidence with a relative precision of $20 \%$, a minimum of 288 antenatal women had to be studied.

All women gave their informed consent prior to their inclusion in the study.

During the study period, 300 women were induced, consented to participate in the study and formed the study group. Their outcomes were compared to women in spontaneous labour.

After delivery, women in both groups (induced and spontaneous), were asked to fill a validated questionnaire containing yes/no, multiple-choice questions and a visual analogue scale. In addition to questions on experience about labour, induced patients were asked about their awareness of induction methods, routes, and the reason for induction, whether they were satisfied with the information provided, their expectation, apprehension and attitude about induction.

The principal author did the majority of the interviews.

Data were entered and analysed using SPSS 16 . The percentage was used to summarize the categorical data. Means \& Standard deviation or Median and Interquartile range was used to summarize the continuous data depending on the skewness. Chi-square test was used to test for associations. An independent t-test was used to compare visual analogue scores.

\section{Results}

The demographic data of the women in both groups is described in Table 10ut of 300 induced women, 196 (65\%) were aware or had prior information about induction.249(84\%)women knew the reason for their induction. 57\% (170) were aware of the benefits of induction of labour, whereas 15\%(45) had been informed about the adverse effects. $57 \%$ (172) were aware of the vaginal route of induction. Only $8 \%$ knew about the intravenous or oral route. (Table 2)

$76 \%$ (227) of women were satisfied with the information provided, felt that time given to decide was adequate, while $27 \%$ (82) observed they were not allowed to discuss enough. $47 \%$ (141) of women thought that induced labour was not natural and 34\% (103) had a feeling that they could have waited for spontaneous labour. 60\% (189) and 63\% (190) felt that induction is for the safety of themselves and their baby respectively.

$40 \%$ (119) women hoped that they would deliver within 6 hours, 31\% (92) felt that they would deliver within 12 hours, and 14\% (44) thought it might go beyond 12 hours. Only 25\% (71) were worried about the duration of labour and $21 \%$ (58) about the mode of delivery. $13 \%$ (37) worried about the discomfort related to induction. $28 \%$ (78) women were apprehensive about the pain and $19 \%$ (53) about the health of the baby.

$61 \%(177)$ had a positive attitude towards labour, whereas $14 \%(40)$ had a negative attitude. $26 \%(75)$ were neutral. 
After delivery, $52 \%$ (156) of women felt labour was more difficult while $29 \%$ (88) felt it was less difficult than expected. $57 \%$ (159) were willing for induction in the subsequent pregnancy and $43 \%$ (122) were not willing for the same.

Analgesia was given on demand, mostly in the latent phase of labour and in the active phase if delivery was not imminent. Injection tramadol was given intramuscularly.

$54 \%(162 / 300)$ of women felt analgesia given was not satisfactory. $42 \%$ (126) demanded better pain relief. When feedback about the process of induction was obtained, $11 \%$ (33) felt oral medication was preferable to vaginal, $21 \%$ (64) wished for a shorter duration of labour, and $10 \%$ (31) felt the number of vaginal examination could be reduced.

$45 \%$ (135) of induced women felt that the process was unbearable. When we studied factors that could have influenced this aspect, only the mode of delivery (vaginal $49 \%$ or caesarean $36 \%$ ) and duration of latent phase greater than 8 hours $(61 \%)$ had an impact of statistical significance ( $p$-value $<0.04$ and 0.04 respectively)(Table 3). Various other factors like induction to delivery interval, parity, duration of latent phase, active phase and second stage, sex of baby, NICU admission and caesarean for failed induction didn't show any statistical significance.

Table 1

Demographic data comparison between spontaneous and Induced group

\begin{tabular}{|lll|}
\hline Variable & Induction & Spontaneous \\
\hline Age $($ Mean \pm SD) & $27( \pm 3)$ & $27( \pm 3)$ \\
\hline Parity $(\mathrm{n})$ & & \\
primi & $217(72 \%)$ & $189(63 \%)$ \\
multi & $83(28 \%)$ & $111(37 \%)$ \\
\hline Period of gestation at delivery (Mean \pm SD) & 39 & 38.4 \\
\hline BMl (Mean \pm SD) & $24.2( \pm 4.6)$ & $23.8( \pm 4.29)$ \\
\hline
\end{tabular}


Table 2

Knowledge and Awareness among the Induced Women

\begin{tabular}{|lll|}
\hline Question & $\begin{array}{l}\text { Number } \mathbf{n} \text { - } \\
\mathbf{3 0 0}\end{array}$ & Percent\% \\
\hline Awareness & 196 & 65.3 \\
Indication & 249 & 83.8 \\
Benefits & 170 & 56.6 \\
Adverse effects & 45 & 15 \\
- Route of & & \\
induction & 26 & 8.6 \\
Oral & 172 & 57.3 \\
Vaginal & 24 & 8 \\
Intravenous & & \\
\hline
\end{tabular}

$71 \%$ of women who needed instrumental delivery felt labour was unbearable compared to $47 \%$ when an instrument was not used, even though this was not statistically significant. $60 \%$ of women with third stage complication felt labour was unbearable compared to $43 \%$ of women who had no complication.

As the second part of the study, we compared the labour experience between induced women and 300 spontaneously labouring women. $68 \%(203)$ in the spontaneous group claimed to have mild to no discomfort compared to $52 \%$ (155) induced women, on the other hand, $32 \%$ (94) women in spontaneous labour felt labour unbearable compared to $45 \%$ (126) induced women which was statistically significant ( $p$ value 0.001 ). Both spontaneous and induced women were satisfied with the support provided during labour that is $87 \%$ and $85 \%$ respectively. Less than half the women in both groups felt that pain relief was satisfactory $45 \%$ (135) in spontaneous and $46 \%$ (138) in the induced group.. Very few women $4 \%(12)$ in spontaneous and $5 \%$ (15) in the induced group felt pain relief was unsatisfactory. (Table 4). 
Table 3

Factors influencing the pain during labour process

\begin{tabular}{|llll|}
\hline Factor & Unbearable & Not unbearable & p-value \\
\hline Latent phase & $77(44 \%)$ & $97(56 \%)$ & 0.04 \\
$>8$ (hrs) & $27(61 \%)$ & $17(39 \%)$ & \\
\hline Mode & & & \\
Vaginal & $97(49 \%)$ & $102(51 \%)$ & 0.04 \\
Caesarean & $36(36 \%)$ & $65(64 \%$ & \\
\hline Instrumental delivery & $10(71 \%)$ & $4(29 \%)$ & 0.078 \\
Yes & $87(47 \%)$ & $98(53 \%)$ & \\
No & & & \\
\hline Third stage complications & $15(60 \%)$ & $10(40 \%)$ & 0.09 \\
Yes & $118(43 \%)$ & $157(57 \%)$ & \\
No & & & \\
\hline
\end{tabular}

Women in both groups were provided with a visual analogue scale to describe their mood during the process of labour.

The parameters considered were whether they were sad or happy, worried or trusting, felt frightened or safe, tired or energetic. While comparing the two, more induced women felt sad $(p<0.001)$ and tired $(p=$ 0.01 ) compared to the spontaneous group. (Table 5) There was no difference between the two groups among the other parameters. 
Table 4

Comparison among Spontaneous and Induced Women

\begin{tabular}{|llll|}
\hline & Spontaneous & Induction & Significance \\
\hline Process of labor & $119(39.6 \%)$ & $39(13 \%)$ & $<0.001$ \\
1. No discomfort & $86(28.8 \%)$ & $126(42 \%)$ & \\
2. Mild discomfort & $95(31.6 \%)$ & $135(45 \%)$ & \\
3. Unbearable & & & \\
Support during labor & $260(87 \%)$ & $257(85 \%)$ & 0.69 \\
1. satisfactory & $24(8 \%)$ & $17(6 \%)$ & \\
2. neutral & $13(4 \%)$ & $12(4 \%)$ & \\
3. unsatisfactory & & & \\
Pain relief & $135(45 \%)$ & $138(46 \%)$ & 0.68 \\
1.satisfactory & $115(51 \%)$ & $147(49 \%)$ & \\
2. not much & $12(4 \%)$ & $15(5 \%)$ & \\
3. unsatisfactory & & & \\
\hline
\end{tabular}


Table 5

Visual analogue scores

\begin{tabular}{|llll|}
\hline VAS & Spontaneous & Induced & Significance \\
\hline $\begin{array}{l}\text { 1.depression to } \\
\text { happy }\end{array}$ & & & $<0.001$ \\
Grade 1 & $29(10 \%)$ & $25(9 \%)$ & \\
Grade 2 & $48(17 \%)$ & $60(21 \%)$ & \\
Grade 3 & $53(19 \%)$ & 114 & \\
Grade 4 & $109(38 \%)$ & $(40 \%)$ & \\
Grade 5 & $47(16 \%)$ & $53(19 \%)$ & \\
\hline $\begin{array}{l}\text { 2. worried to } \\
\text { trusting }\end{array}$ & & $29(11 \%)$ & \\
Grade 1 & $25(9 \%)$ & $27(10 \%)$ & \\
Grade 2 & $59(20 \%)$ & $68(24 \%)$ & \\
Grade 3 & $68(24 \%)$ & $51(18 \%)$ & \\
Grade 4 & $95(33 \%)$ & $84(30 \%)$ & \\
Grade 5 & $41(14 \%)$ & $50(18 \%)$ & \\
\hline 3.frightened to & & & \\
safe & & & \\
Grade 1 & $37(13 \%)$ & $28(10 \%)$ & \\
Grade 2 & $68(24 \%)$ & $79(28 \%)$ & \\
Grade 3 & $60(21 \%)$ & $64(23 \%)$ & \\
Grade 4 & $80(28 \%)$ & $68(24 \%)$ & \\
Grade 5 & $39(14 \%)$ & $41(15 \%)$ & \\
\hline $\begin{array}{l}\text { tired to } \\
\text { energetic }\end{array}$ & & & \\
Grade 1 & & & \\
Grade 2 & & & \\
Grade 3 & & & \\
Grade 4 & & & \\
Grade 5 & & & \\
\end{tabular}




\section{Knowledge/information.}

Labour induction has become an indispensable obstetric procedure, the rates of which are on a rising trend. Our induction rate is $34 \%$ and we found in our study that though $65 \%$ were aware of what induction was, and $85 \%$ knew the indication, only $15 \%$ were aware of the risks, very few about $8 \%$ knew about the intravenous routes of administration, very much indicative of lack of information. Similarly, Moore et al (Moore et al., 2014)., in their study showed induced women were aware of the benefits but not about risks, in contrast when women were given information leaflets before the procedure they had an understanding of side effects of prostaglandins (Cooper \& Warland, 2011). In a German study, 60\% wanted more information on alternatives to induction and $55 \%$ more information on the drugs used (König-Bachmann et al., 2017). The frustration among the patients regarding the lack of information is palpable in many studies (Enabor et al., 2012) (Brown \& Furber, 2015) (Shetty et al., 2005) Inadequate information, in turn, increases the anxiety levels (Brown \& Furber, 2015). Women wanted information which prepared them for the realities of labour (Beake, RM, MA, Research Associate et al., 2018). In one study patients reported not having received any information on IOL from any health profession (Murtagh \& Folan, 2014).

Hence it is important to disperse honest information to the patients on every aspect of induction so that they have a vivid picture of what to expect during labour.

Though everything is documented on the consent sheet it is equally important to make sure they understand the pros and cons before signing the same to ensure informed consent.

\section{Decision making:}

About three-fourth were satisfied with the information, discussion and time they were given to make a decision. Not many were aware of the time taken for delivery. When medical interventions are indicated it is of paramount importance to involve the women and her family in the process of decision making. We need to encourage them to ask questions and give them ample time to decide. Although induction is a routine procedure for obstetricians, for a pregnant woman it is new or unexpected, so an opportunity for discussion is a must.

Induction of labour was presented as an inevitable next step in one study (Moore et al., 2014) and women should know about the IOL process, medications, risks, and options as part of the decision-making process. Though $75 \%$ of women were satisfied with the time given to decide, the reality is that most of our women do not understand the whole process of induction. Some women fear the mention of the word induction because a sense of failure of not being able to go into labour spontaneously sets in.

In Indian culture, it is believed that the good fortune of the baby is dependent on its date and time of birth. Many women may not prefer to deliver on certain days and this needs to be respected after due consideration about the maternal and foetal well-being. Family members are always involved in decision making 
Molenaar et al (Molenaar et al., 2018), reported that it is important that both partners are involved in shared decision making. In Ireland, there was a feeling in induced women that they were forced to decide on induction or strongly consider it (Murtagh \& Folan, 2014). In our study, some women and their companions entrusted this decision on us because they believed whatever we do is in the best interest of both the mother and the newborn.. This belief and trust was similarly noted in studies done by (KönigBachmann et al., 2017) Women cited trust as the most important element in their agreement to be induced (Moore et al., 2014)

\section{Attitude and expectations:}

The negative attitude seen in $40 \%$ of the women was mostly because they perceived induction to be unnatural. This changed their concept of childbirth and caused a shift of their expectations as highlighted by Gatward et al., (Gatward et al., 2010) It was reassuring to know that not many were apprehensive regarding the health of the baby or themselves. According to Shetty et al., the induction to delivery interval has a bearing on women's attitude towards induction. (Shetty et al., 2005) More than half of the women found labour more difficult compared to their expectation and were not satisfied with the pain relief provided. The pain relief given was injection Tramadol (opioid analgesic) and breathing relaxation techniques taught by trained nursing staff. We do not have provision for epidural, however, only a negligible number of women asked for it. Prolonged latent phase, instrumental delivery, third stage complications like perineal tears, manual removal of placenta were a few factors that had an influence on pain and made it unbearable.

\section{Care and support.}

Women require continuous support during childbirth. Support from health care persons plays a pivotal role in improving women experience in induction. (Warren et al., 2018)(Ford et al., 2009). It is recommended by WHO that labouring woman should have a companion of her choice during the entire process of childbirth. Though support from family members plays a significant role, in our hospital family members are not allowed inside the labour room due to a dearth of space and privacy, and also because of increased risk of infection. One major positive aspect of our study was the majority (85\%) were extremely happy and satisfied with the care and support that they received from doctors and nursing staff. In contrast to our findings women were unhappy with the response of midwives and felt neglected (Coates et al., 2019).

Labour is a stressful event for many women filled with fear and anxiety; the presence of a healthcare professional may reduce her anxiety and boost her morale to a large extent and also provide nonpharmacological pain relief.

\section{Induced versus Spontaneous labour:}

Induced women felt more discomfort during the entire process than spontaneous labour $(55 \%$ vs $32 \% \mathrm{P}=$ 0.001 ), mode of delivery and latent phase had an impact of statistical significance ( $p$ value $<0.04$ and 0.04 respectively) for obvious reasons. 
While comparing the mood of the women in the two groups using the visual analogue scale, induced women were sadder $(p<0.001)$ and tired $(p=0.01)$ compared to the spontaneous group which maybe because of the increased time taken, operative interference and a feeling of non-accomplishment of spontaneous vaginal delivery. There was no difference between the pain relief as an equal number of women in both groups felt pain relief was unsatisfactory. On the contrary, in Sweden women with spontaneous labour experienced more pain than induced women but the induced women had used more of epidural analgesia. (Hildingsson et al., 2011) Henderson et al. reported that induced women were more worried and were not happy with the staff caring for them during labour (Coates et al., 2019). More than half of our women in the induced group were ready to undergo induction in the next pregnancy (compared to $74 \%$ in a study by Heimsted et al.,) (Heimstad et al., 2007), however, they preferred a drug which would act faster and can be orally taken.

\section{Limitations:}

We had a fixed set of questions and answers.

This survey was done after delivery.

\section{Conclusion}

Induced women were not well informed about adverse effects and felt labour was unbearable. Induced women were more tired and sad at the end of labour. It is important to enhance their knowledge by providing complete unbiased and realistic information on all aspects of induction and also encourage women to take part in decision making. Women should be informed about the local facilities and induction protocols. Local protocols to provide adequate analgesia should be implemented to alleviate the fear of pain.

Good counselling and compassionate care from healthcare workers is the need of the hour to increase the satisfaction levels of induced women and achieving a goal of the positive childbirth experience.

\section{Abbreviations}

$\mathrm{IOL}$ induction of labour

NICU Neonatal intensive care unit

\section{Declarations}

1. Ethical Clearance was obtained from Institutional Ethical Clearance Committee (MUEC).( Copy attached in supplementary material).

2. The consent to publish was taken from patients at the time of enrolling .

3. The data will be available from corresponding author on request. 
4. No Competing Interest

5. Me and my coauthors hereby declare that our study was not funded by any source.

6.Authors contributions are attached in supplementary information.

7. Acknowledgement. We would like to thank DR Ashalatha Shetty Department of Obstetrics and Gynaecology, Aberdeen Maternity Hospital, Foresterhill, Aberdeen AB25 2ZL, Scotland, for her help in formatting the questionnarre and Dr Asha Kamat ,Department of Statistics ,KMC ,Manipal for her help in statistics.

\section{References}

1. Beake RM, Research Associate MA, Chang S, MPhil BA, Lecturer PhD, Cheyne YS, MSc RM,RGN, PhD, Professor of Midwifery, Spiby H, MPhil RN, RM, Professor of Midwifery, Sandall H. RM, MSc PhD, Professor of Social Science and Women's Health J, Bick D. (2018). Experiences of early labour management from perspectives of women, labour companions and health professionals: A systematic review of qualitative evidence. In Midwifery. https://doi.org/10.1016/j.midw.2017.11.002.

2. Brown SJS, Furber CM. Women's experiences of cervical ripening as inpatients on an antenatal ward. Sexual Reproductive Healthcare. 2015. https://doi.org/10.1016/j.srhc.2015.06.003.

3. Coates R, Cupples G, Scamell A, McCourt C. (2019). Women's experiences of induction of labour: Qualitative systematic review and thematic synthesis. In Midwifery. https://doi.org/10.1016/j.midw.2018.10.013.

4. Cooper M, Warland J. Improving women's knowledge of prostaglandin induction of labour through the use of information brochures: A quasi-experimental study. Women Birth. 2011. https://doi.org/10.1016/j.wombi.2010.10.003.

5. Enabor OO, Olayemi OO, Bello FA, Adedokun BO. Cervical ripening and induction of labour-awareness, knowledge and perception of antenatal attendees in Ibadan, Nigeria. J Obstet Gynaecol. 2012. https://doi.org/10.3109/01443615.2012.657271.

6. Ford E, Ayers S, Wright DB. Measurement of maternal perceptions of Support and Control in Birth (SCIB). Journal of Women's Health. 2009. https://doi.org/10.1089/jwh.2008.0882.

7. Gatward H, Simpson M, Woodhart L, Stainton MC. Women's experiences of being induced for postdate pregnancy. Women Birth. 2010. https://doi.org/10.1016/j.wombi.2009.06.002.

8. Heimstad R, Romundstad PR, Hyett J, Mattsson L, Salvesen K Å. (2007). Women's experiences and attitudes towards expectant management and induction of labor for post-term pregnancy. Acta Obstetricia et Gynecologica Scandinavica. https://doi.org/10.1080/00016340701416929.

9. Hildingsson I, Karlström A, Nystedt A. Women's experiences of induction of labour - Findings from a Swedish regional study. Aust N Z J Obstet Gynaecol. 2011. https://doi.org/10.1111/j.1479828X.2010.01262.x. 
10. König-Bachmann M, Schwarz C, Zenzmaier C. Women's experiences and perceptions of induction of labour: Results from a German online-survey. European Journal of Midwifery. 2017. https://doi.org/10.18332/ ejm/76511.

11. Leduc D, Biringer A, Lee L, Dy J, Corbett T, Duperron L, Lange I, Muise S, Parish B, Regush L, Wilson K, Yeung G, Crane J, Gagnon R, Sawchuck D, Senikas V. Induction of Labour. Journal of Obstetrics Gynaecology Canada. 2013. https://doi.org/10.1016/S1701-2163(15)30842-2.

12. Molenaar J, Korstjens I, Hendrix M, de Vries R, Nieuwenhuijze M. Needs of parents and professionals to improve shared decision-making in interprofessional maternity care practice: A qualitative study. Birth. 2018. https://doi.org/10.1111/birt.12379.

13. Moore JE, Low LK, Titler MG, Dalton VK, Sampselle CM. Moving toward patient-centred care: Women's decisions, perceptions, and experiences of the induction of labor process. Birth. 2014. https://doi.org/10.1111/birt.12080.

14. Murtagh M, Folan M. Women's experiences of induction of labour for post-date pregnancy. British Journal of Midwifery. 2014. https://doi.org/10.12968/bjom.2014.22.2.105.

15. NICE Clinical Guideline 70. (2008). Inducing labour. In National Institute for Health and Care Execellence.

16. Shetty A, Burt R, Rice P, Templeton A. Women's perceptions, expectations and satisfaction with induced labour - A questionnaire-based study. European Journal of Obstetrics Gynecology Reproductive Biology. 2005. https://doi.org/10.1016/j.ejogrb.2005.03.004.

17. Thompson R, Miller YD. Birth control: To what extent do women report being informed and involved in decisions about pregnancy and birth procedures? BMC Pregnancy Childbirth. 2014. https://doi.org/10.1186/1471-2393-1462.

18. Warren, C. E., Njue, R., Ndwiga, C., Abuya, T., Kabo, J. W., SuellenMilleraAndreLalonde,McMahon, S. A., George, A. S., Chebet, J. J., Mosha, I. H., Mpembeni, R. N. M., Winch,P. J., Kendall, T., Downe, S., World Health Organization, Wesson, J., Hamunime, N.,Viadro, C., Carlough, M., ... Access, O. (2018). WHO recommendations: Intrapartum care for a positive childbirth experience. BMC Pregnancy and Childbirth. https://doi.org/10.5935/1676 4285.20155067.

\section{Supplementary Files}

This is a list of supplementary files associated with this preprint. Click to download.

- FLOWCHART.docX

- STROBESUBMISSION.doc 